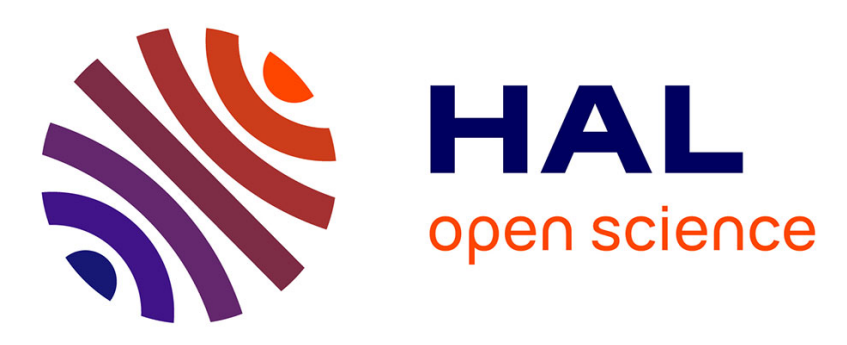

\title{
Edge detection and image restoration with anisotropic topological gradient
}

\author{
Stanislas Larnier, Jérôme Fehrenbach
}

\section{To cite this version:}

Stanislas Larnier, Jérôme Fehrenbach. Edge detection and image restoration with anisotropic topological gradient. ICASSP 2010: International Conference on Acoustics, Speech, and Signal Processing, Mar 2010, Dallas, United States. pp.1362-1365, 10.1109/ICASSP.2010.5495448 . hal-01333753

\section{HAL Id: hal-01333753 https://hal.science/hal-01333753}

Submitted on 19 Jun 2016

HAL is a multi-disciplinary open access archive for the deposit and dissemination of scientific research documents, whether they are published or not. The documents may come from teaching and research institutions in France or abroad, or from public or private research centers.
L'archive ouverte pluridisciplinaire HAL, est destinée au dépôt et à la diffusion de documents scientifiques de niveau recherche, publiés ou non, émanant des établissements d'enseignement et de recherche français ou étrangers, des laboratoires publics ou privés. 


\title{
EDGE DETECTION AND IMAGE RESTORATION WITH ANISOTROPIC TOPOLOGICAL GRADIENT
}

\author{
Stanislas Larnier, Jérôme Fehrenbach \\ Institut de Mathématiques de Toulouse, \\ Université Paul Sabatier, 31062 Toulouse cedex 9, France. \\ e-mail: $\{$ stanislas.larnier, jerome.fehrenbach\}@math.univ-toulouse.fr
}

\begin{abstract}
Topological asymptotic analysis provides tools to detect edges and their orientation. The purpose of this article is to show the possibilities of anisotropic topological gradient in image restoration. Previous methods based on the topological gradient used isotropic diffusion to restore images. These methods are improved here by using anisotropic diffusion and differentiating between principal and secondary edges. A texture detector is also used to increase the diffusion outside textured regions. Numerical results are presented, including a comparison with the Non-Local Means method. The algorithms presented here lead to results similar to the Non-Local Means (in terms of quality), and shorter processing times.
\end{abstract}

Index Terms - Edge detection, topological gradient, image restoration, anisotropic diffusion.

\section{INTRODUCTION}

A classical way to restore an image $u$ from its noisy version $v$ defined in a domain $\Omega \subset \mathbb{R}^{2}$ is to solve the following PDE problem

$$
\left\{\begin{aligned}
-\nabla \cdot(c \nabla u)+u & =v \text { in } \Omega, \\
\partial_{n} u & =0 \text { on } \partial \Omega,
\end{aligned}\right.
$$

where $c$ is a positive constant or a tensor [1] and $\partial_{n}$ denotes the normal derivative to $\partial \Omega$.

Topological optimization provides tools to detect cracks [2]. These tools were adapted to detect edges [3]. An image can be viewed as a piecewise smooth function and edges can be considered as its set of singularities. A major advantage of topological gradient is the computing time. Only $O(N \cdot \log (N))$ operations are needed to solve the image processing problem, where $N$ is the number of pixels.

The topological gradient can also be used in image edge analysis, image classification, inpainting problems and in segmentation $[3,4,5]$. This technique can be applied to gray level and color images, but also to three-dimensional images, or movies.

In the work [3], $c$ is an isotropic tensor taking only two values, $c_{0}$ in the smooth part of the image and a small value $\varepsilon$ on edges. The objective of the present work is to take advantage of additional informations provided by topological asymptotic analysis: the edge orientation and jump amplitude. This allows to define the tensor $c$ in a way more adapted to the image content. An anisotropic restoration algorithm is presented. This simple algorithm is compared to the previous isotropic restoration algorithm and to the Non-Local Means method.

In section 2, we recall the topological gradient method for image restoration. Our restoration algorithms are presented in section 3. In section 4, we present numerical results in image restoration and some comparisons.

\section{TOPOLOGICAL GRADIENT FORMULATION}

Let $\Omega$ be an open bounded domain of $\mathbb{R}^{2}$ and $v$ a given function in $L^{2}(\Omega)$. The image restoration problem is defined on the domain and reads as follows: find $u \in H^{1}(\Omega)$ such that

$$
\left\{\begin{aligned}
-\nabla \cdot(c \nabla u)+u & =v \text { in } \Omega \\
\partial_{n} u & =0 \text { on } \partial \Omega .
\end{aligned}\right.
$$

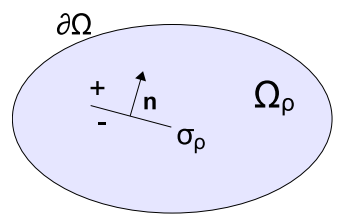

Fig. 1. Cracked domain.

At a given point $x_{0} \in \Omega$, we insert a small insulating crack $\sigma_{\rho}=x_{0}+\rho \sigma(n)$ where $\sigma(n)$ is a straight crack and $n$ is a unit vector normal to the crack. The perturbed domain created by the insertion of this crack is $\Omega_{\rho}=\Omega \backslash \sigma_{\rho}$. The perturbed solution $u_{\rho} \in H^{1}\left(\Omega_{\rho}\right)$ satisfies

$$
\left\{\begin{aligned}
-\nabla \cdot\left(c \nabla u_{\rho}\right)+u_{\rho} & =v \text { in } \Omega_{\rho}, \\
\partial_{n} u_{\rho} & =0 \text { on } \partial \Omega, \\
\partial_{n} u_{\rho} & =0 \text { on } \sigma_{\rho} .
\end{aligned}\right.
$$



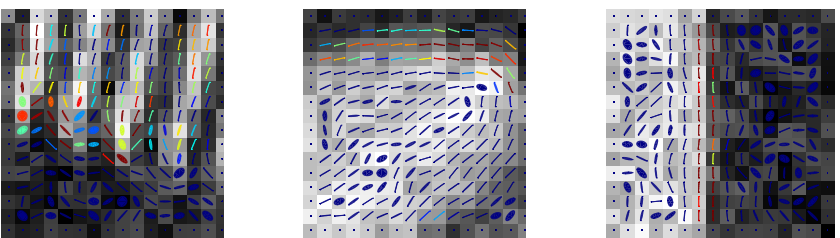

Fig. 2. Ellipse representation: parts of noisy Barbara image 3. From left to right, scarf, books and table.

Our edge detection method consists in looking for a crack $\sigma$ such that the energy $j(\rho)=J_{\rho}\left(u_{\rho}\right)=\frac{1}{2} \int_{\Omega_{\rho}}\left\|\nabla u_{\rho}\right\|^{2}$ is small. In [2], it is proved that the cost function $j$ has the following asymptotic expansion

$$
j(\rho)-j(0)=\rho^{2} g\left(x_{0}, n\right)+o\left(\rho^{2}\right),
$$

where the topological gradient $g$ is given by

$$
g\left(x_{0}, n\right)=-\pi c\left(\nabla u\left(x_{0}\right) . n\right)\left(\nabla p\left(x_{0}\right) . n\right)-\pi\left|\nabla u\left(x_{0}\right) . n\right|^{2},
$$

and $p_{0}$ is the solution to the adjoint problem:

$$
\left\{\begin{aligned}
-\nabla \cdot\left(c \nabla p_{0}\right)+p_{0} & =-\partial_{u} J_{0}(u) & & \text { in } \Omega, \\
\partial_{n} p_{0} & =0 & & \text { on } \partial \Omega .
\end{aligned}\right.
$$

The topological gradient can be written as $g(x, n)=$ $n^{T} M(x) n$, where $M(x)$ is the $2 \times 2$ symmetric matrix defined by

$$
\begin{gathered}
M(x)=-\pi c \frac{\nabla u_{0}(x) \nabla p_{0}(x)^{T}+\nabla p_{0}(x) \nabla u_{0}(x)^{T}}{2} \\
-\pi \nabla u_{0}(x) \nabla u_{0}(x)^{T} .
\end{gathered}
$$

For a given $x, g(x, n)$ takes its minimal value when $n$ is the eigenvector associated to the lowest eigenvalue $\lambda_{\min }(x)$ of $M(x)$. This value is the topological gradient associated to the optimal orientation of the crack $\sigma_{\rho}$.

The contours are located at points $x$ where $\lambda_{\min }(x)$ is the most negative and their orientation is given by the corresponding eigenvector.

Figure 2 shows parts of noisy Barbara image with a uniform additive noise. The ellipses represent the topological gradient tensor $M(x)$ at each pixel of the image. The edge orientation is given by the associated eigenvector. Note that the ellipses are oriented in the same direction as the texture edges.

\section{ALGORITHMS FOR IMAGE RESTORATION}

\subsection{Isotropic topological gradient}

Let us first recall the algorithm for isotropic topological gradient presented in [3]. It consists in inserting small
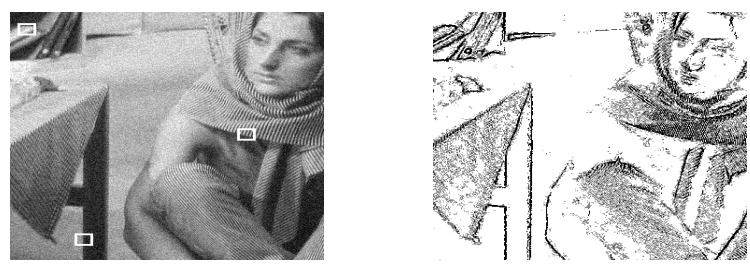

Fig. 3. Noisy image of Barbara and detected edges.

heterogeneities in regions where the topological gradient is smaller than a given threshold $\delta<0$ (typical value : $\delta \in[-1500,-250])$.

- Initialization: $c=c_{0}$.

- Computation of $u_{0}$ and $p_{0}$, solutions of direct (2) and adjoint (6) problems.

- Computation of the $2 \times 2$ matrix $M(x)$ and its eigenvalues at each point of the domain.

- Set $c(x)= \begin{cases}c_{0} / 10 & \text { if } x \in \Omega \text { and } \lambda_{\min }(x)<\delta \\ c_{0} & \text { otherwise }\end{cases}$

- Computation of $u$ solution to problem (2).

\subsection{Anisotropic topological gradient}

It is well known that the diffusion equation (1) can lead to better restoration results if $c$ is defined as an anisotropic tensor: it should be elongated in the edges direction. A diffusion coefficient $c_{1}<<1$ is used in the direction given by the eigenvector associated to $\lambda_{\min }$.

- Initialization: $c=c_{0}$.

- Computation of the solutions $u_{0}$ and $p_{0}$ of the direct (2) and adjoint (6) problems.

- Computation of the eigenvalue decomposition of $M$ as $M(x)=\left(P D P^{-1}\right)(x)$.

- Extraction of the valleys of $\lambda_{\text {min }}$ : they represent the edges.

- Set

$$
\begin{gathered}
c(x)=P(x)\left(\begin{array}{cc}
\phi(x) & 0 \\
0 & c_{0}
\end{array}\right) P^{-1}(x) \\
\phi(x)= \begin{cases}c_{1}\left(\lambda_{\min }(x)\right) & \text { if } x \in \Omega, \lambda_{\min }(x)<\delta \\
& \text { and } x \text { is in a valley, } \\
c_{0} & \text { otherwise }\end{cases}
\end{gathered}
$$

- Computation of $u$ solution of (2) with the new $c$.

\subsection{Secondary edges and smoothing}

Texture may contain edges with smaller amplitude. We call them secondary edges. To take these into account, we use a second threshold $\delta^{\prime}>\delta$. In the definition of $\phi$ (equation (8)), we add the condition:

$\phi(x)=c_{2}\left(\lambda_{\min }(x)\right)$ if $\delta<\lambda_{\min }(x)<\delta^{\prime}$ and $x$ is in a valley. 


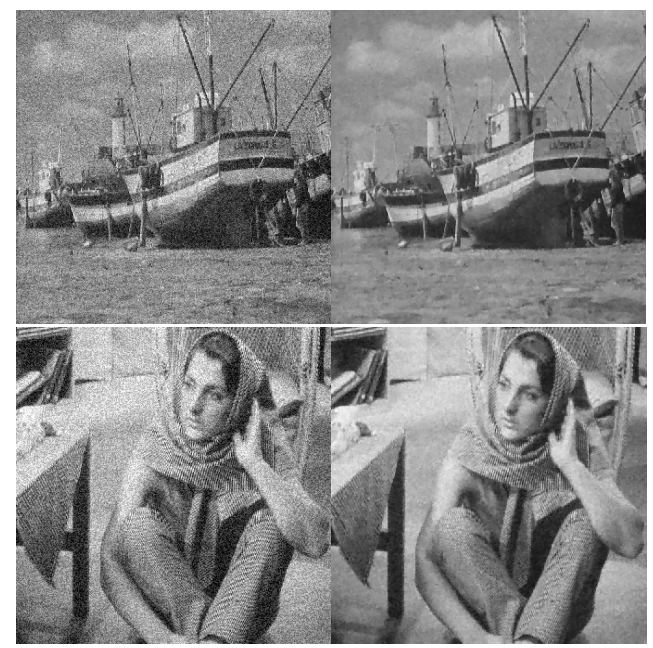

Fig. 4. Noisy and ATGS+ restoration of boat and Barbara images

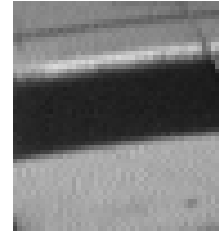

(a) Original

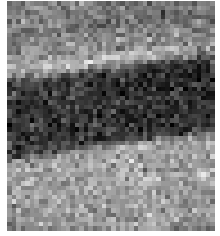

(b) Noisy

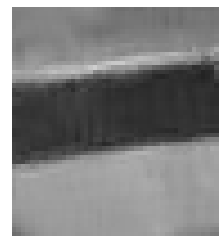

(e) NLM (c) ITG (d) ATGS+

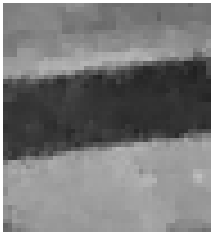

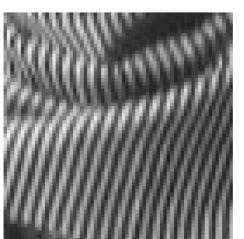

(a) Original

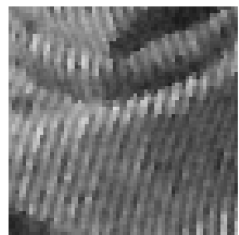

(c) ITG

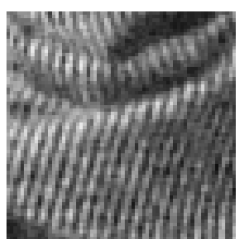

(d) ATGS+

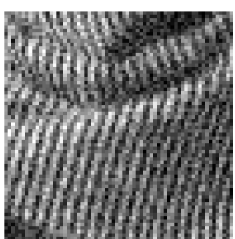

(b) Noisy

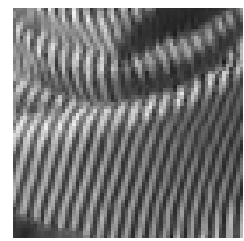

(e) NLM
Fig. 6. Parts of original Barbara image, noisy one, ITG, ATGS+ and NLM restoration.

\begin{tabular}{|c|c|c||c||c|c|c|}
\multicolumn{3}{c||}{ Boat } & \multicolumn{1}{c||}{} & \multicolumn{3}{c|}{ Barbara } \\
PSNR & SSIM & Time & & PSNR & SSIM & Time \\
\hline 22,53 & 0,444 & $\#$ & Noisy & 19,85 & 0,374 & $\#$ \\
\hline 26,93 & 0,721 & $4,48 \mathrm{~s}$ & ITG & 25,36 & 0,644 & $4,40 \mathrm{~s}$ \\
\hline 27,01 & 0,723 & $4,78 \mathrm{~s}$ & ITGS & 25,46 & 0,655 & $5,25 \mathrm{~s}$ \\
\hline 27,27 & 0,764 & $6,20 \mathrm{~s}$ & ITGS+ & 25,93 & 0,751 & $6,12 \mathrm{~s}$ \\
\hline 26,99 & 0,722 & $4,52 \mathrm{~s}$ & ATG & 25,41 & 0,646 & $4,45 \mathrm{~s}$ \\
\hline 27,09 & 0,724 & $5,18 \mathrm{~s}$ & ATGS & 25,78 & 0,663 & $5,22 \mathrm{~s}$ \\
\hline 27,38 & 0,764 & $6,25 \mathrm{~s}$ & ATGS+ & 26,27 & 0,758 & $6,28 \mathrm{~s}$ \\
\hline 27,72 & 0,772 & $13,1 \mathrm{~s}$ & NLM & 25,23 & 0,789 & $36,5 \mathrm{~s}$ \\
\hline
\end{tabular}

Table 1. Boat and Barbara results.

We compare quantitatively the different restorations on gray level images using PSNR (Peak Signal to Noise Ratio) expressed in $\mathrm{dB}$ and the SSIM (Structural SIMilarity) [9]. We give the CPU time in seconds for each algorithm.

Table 1 presents the results on two images: boat $(512 \times$ $512)$ and Barbara $(512 \times 512)$ see Figure 4 . The noise used for the boat image is a Gaussian additive noise. The noise used for Barbara image is an additive noise following a uniform distribution.

Figure 5 (c) and (d) compare the results of ITG and ATGS+ restoration. The restoration of contours is more accurate using anisotropic restoration, although the quantitative estimators do not reflect this improvement (see Table 1).

Figure 6 (c) and (d) compare the results of ITG and ATGS+ restoration. The aim is to show that the texture is better reconstructed in the new restoration. Adding anisotropy and secondary edges clearly improves the results on Barbara image (see Table 1).

A comparative study with the NLM was performed. With a Gaussian noise, we obtained similar results for images which are not composed of self similar textures. On Barbara image corrupted with Gaussian additive noise, the results provided by the NLM outperform our diffusion method. 


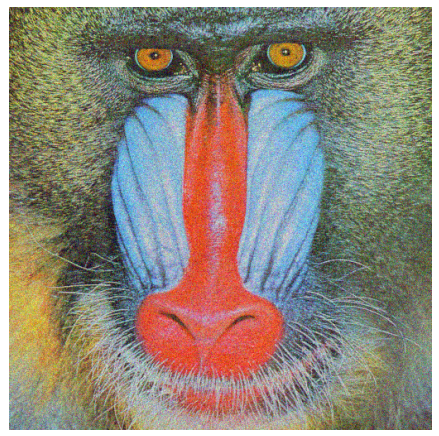

Fig. 7. Noisy Mandrill image.

\begin{tabular}{c||c|c|c|}
\multicolumn{1}{c||}{} & \multicolumn{3}{c}{ Mandrill } \\
& PSNR & SSIM & CPU \\
\hline Noisy & 20,16 & 0,552 & $\#$ \\
\hline ATGS+ & 22,79 & 0,698 & $17,2 \mathrm{~s}$ \\
\hline NLM & 22,63 & 0,708 & $29,6 \mathrm{~s}$ \\
\hline
\end{tabular}

Table 2. Mandrill results.

This is sensible since in the NLM algorithm, the denoised value at a pixel $x$, is a mean of the values of all pixels whose Gaussian neighborhood looks like the neighborhood of $x$. So the efficiency on a self similar image was predictable.

Figure 7 shows the noisy Mandrill $(512 \times 512 \times 3)$ color image. The three channels (RGB) were treated separately. The NLM and ATGS+ restoration provide similar results in terms of quality (see Table 2 and Figure 8 (c) and (d)). ATGS+ is faster (see Table 2).

\section{CONCLUSION}

An algorithm was presented to take into account the orientation of the edges detected by topological asymptotic analysis. The diffusion tensor used to restore an image is anisotropic on the edges, with a small diffusion coefficient on primary edges and a larger one on the secondary edges. This algorithm was compared to the Non-Local Means method. Similar performances were obtained for smaller computing times.

\section{REFERENCES}

[1] G. Aubert and P. Kornprobst, Mathematical Problems in Image Processing: Partial Differential Equations and the Calculus of Variations, vol. 147, Springer-Verlag, Applied Mathematical Sciences, November 2001.

[2] S. Amstutz, I. Horchani, and M. Masmoudi, "Crack detection by the topological gradient method," Control and Cybernetics, vol. 34, no. 1, pp. 81-101, 2005.

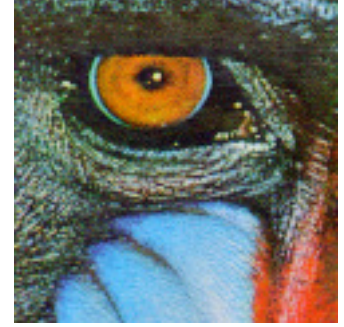

(a) Original

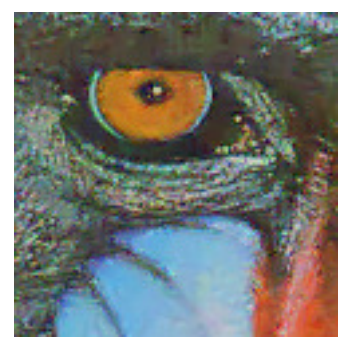

(c) NLM restoration

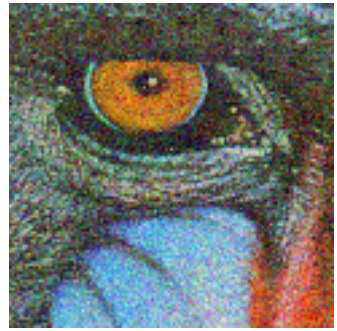

(b) Noisy

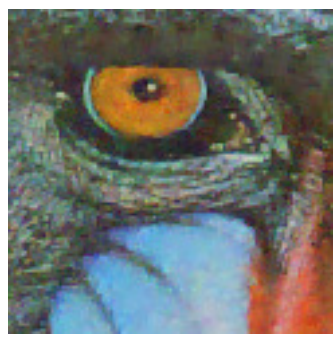

(d) ATGS+ restoration
Fig. 8. Parts of original mandrill image, noisy one, NLM and ATGS+ restoration.

[3] L. J. Belaid, M. Jaoua, M. Masmoudi, and L. Siala, "Image restoration and edge detection by topological asymptotic expansion," C. R. Acad. Sci. Paris, vol. 342, issue 5, pp. 313-318, March 2006.

[4] D. Auroux and M. Masmoudi, "A one-shot inpainting algorithm based on the topological asymptotic analysis," Computational and Applied Mathematics, vol. 25, no. 23, pp. 251-267, 2006.

[5] D. Auroux and M. Masmoudi, "Image processing by topological asymptotic expansion," J. Math. Imaging Vision, vol. 33, issue 2, pp. 122-134, February 2009.

[6] A. T. Targhi and A. Shademan, "Clustering of singular value decomposition of image data with applications to texture classification," Proc. SPIE, vol. 5150, pp. 972979, April 2003.

[7] A. Buades, B. Coll, and J.M Morel, "A review of image denoising algorithms, with a new one," Multiscale Modeling and Simulation (SIAM interdisciplinary journal), vol. 4, issue 2, pp. 490-530, 2005.

[8] G. Peyre, "Non-local means toolbox," http://www. mathworks.fr/matlabcentral/fileexchange/13619.

[9] Z. Wang, A. C. Bovik, H. R. Sheikh, and E. P. Simoncelli, "Image quality assessment : From error visibility to structural similarity," IEEE Transactions on Image Processing, vol. 13, no. 4, pp. 600-612, April 2004. 